\title{
Prática Baseada em Evidências em Psicologia e Idosos: Conceitos, Estudos e Perspectivas
}

\author{
Thiago Vinicius Monteleone \\ Universidade São Judas Tadeu, SP, Brasil.
}

\author{
Carla Witter \\ Universidade São Judas Tadeu, SP, Brasil.
}

Resumo: O presente artigo teve por objetivo apresentar o desenvolvimento histórico do movimento da Prática Baseada em Evidências em Psicologia, bem como a integração deste modelo à atuação psicoterápica junto à população idosa. O conceito de prática baseada em evidências é um dos temas que tem sido amplamente discutido na literatura internacional da Psicologia. Nestas discussões, com poucas exceções, compreende-se que a relevância da prática psicoterápica depende intimamente de evidências que indiquem o resultado de suas intervenções. Com a população idosa não deveria ser diferente, contudo, apesar do aumento da procura por serviços clínicos por parte desta faixa-etária, o processo psicoterápico com idosos ainda é pouco discutido pelos profissionais da Psicologia, o que tem produzido incertezas e pouca confiabilidade nas intervenções realizadas. Deste modo, o artigo apresenta o desenvolvimento histórico do conceito de Prática Baseada em Evidências em Psicologia, as influências científicas, clínicas e de associações de classes neste desenvolvimento, bem como a integração deste modelo à prática clínica com a população idosa, demonstrando-se os principais estudos existentes e as perspectivas para a obtenção de evidências empíricas do resultado psicoterápico para esta população.

Palavras-chave: Psicologia Clínica, Envelhecimento, Psicoterapia.

\section{Evidence-Based Practice in Psychology and the Elderly: Concepts, Studies and Perspectives}

\begin{abstract}
This article aims to present the historical development of the movement of Evidence-Based Practice in Psychology as well as the integration of this model with the psychotherapeutic performance with the elderly. The concept of evidence-based practice is one of the themes that have been more widely discussed in the international literature of psychology. In these discussions, with few exceptions, it is understood that the relevance of psychotherapeutic practice depends intimately of evidence indicating the results of its interventions. With the elderly population should not be different; however, despite the increasing demand for clinical services by this age group, the psychotherapeutic process with elderly people is still little discussed by psychology professionals, which has produced uncertainty and unreliability in interventions. Thus, the article presents the historical development of the concept of evidence-based practice in psychology, the scientific influences, clinical and professional associations in this development, as well as the integration of this model to clinical practice with the elderly, demonstrating the main existing studies and prospects for obtaining empirical evidence of the psychotherapy outcome for this population.
\end{abstract}

Keywords: Clinical Psychology, Aging, Psychotherapy. 


\title{
La práctica basada en la evidencia en Psicología y los adultos mayores: Conceptos, Estudios y Perspectivas
}

\begin{abstract}
Resumen: Este artículo tuvo como objetivo presentar la evolución histórica del movimiento de la práctica basada en la evidencia en Psicología, así como la integración de este modelo con la actuación psicoterapéutica con los adultos mayores. El concepto de la práctica basada en la evidencia es uno de los temas que ha sido ampliamente discutido en la literatura internacional de la psicología. En estas discusiones, con pocas excepciones, se entiende que la importancia de la práctica psicoterapéutica depende íntimamente de las evidencias que indican los resultados de sus intervenciones. Con la población de edad avanzada no debería ser diferente, sin embargo, a pesar de la mayor demanda de servicios clínicos de este grupo de edad, el proceso psicoterapéutico con personas mayores es todavía poco discutido por profesionales de la psicología, lo que ha producido incertidumbre y falta de fiabilidad en las intervenciones. Así, el artículo presenta el desarrollo histórico del concepto de la práctica basada en la evidencia en psicología, las influencias científicas, profesionales y de asociaciones en este desarrollo, asî como la integración de este modelo para la práctica clínica con los adultos mayores, lo que demuestra los principales estudios y perspectivas de obtener evidencia empírica del resultado de la psicoterapia para esta población.
\end{abstract}

Palabras clave: Psicología Clínica, Envejecimiento, Psicoterapia.

\section{Introdução}

O movimento de prática baseada em evidências é, atualmente, um dos temas que mais tem produzido discussões na literatura internacional da Psicologia. Neste contexto, o debate sobre o tipo de intervenção psicológica para determinadas queixas tem modificado o modo tradicional de escolha do tratamento, não considerando apenas a predileção e a experiência profissional, mas somando-se a estes critérios as pesquisas existentes e as idiossincrasias dos pacientes.

Iniciado em meio a um intenso debate entre pesquisadores e clínicos na década de 1950, o movimento de Prática Baseada em Evidências ainda produz controvérsias e acalorados conflitos na literatura internacional da Psicologia. Nestas discussões, salvo algumas exceções, compreende-se que a relevância da prática psicoterápica depende intimamente de evidências que indiquem o resultado de suas intervenções.

Com a população idosa não deveria ser diferente. Contudo, apesar do aumento da procura por serviços clínicos por parte desta faixa-etária, devido às mudanças demográficas que ocorrem mundialmente, o processo psicoterapêutico com idosos é, ainda, pouco discutido pelos profissionais da Psicologia, o que tem produzido incertezas e pouca confiabilidade nas intervenções realizadas. Tal fato demonstra um atraso no desenvolvimento de intervenções psicoterápicas com esta população, se comparados às metodologias de intervenção produzidas em outras áreas comumente utilizados por esta população (por exemplo, medicina, enfermagem, fisioterapia etc.).

Desse modo, o presente artigo visa apresentar o desenvolvimento histórico do conceito de Prática Baseada em Evidências em Psicologia, as influências científicas, clínicas e de associações de classes nesse desenvolvimento, bem como a integração deste modelo à prática clínica com a população idosa, apresentando os principais estudos existentes e as perspectivas para a obtenção de evidências empíricas do resultado psicoterápico para esta população.

\section{Prática baseada em evidências em Psicologia: desenvolvimento histórico}

Apesar do movimento que sugere que os profissionais da Psicologia devam, dentre outras recomendações, pautar sua prática psicoterápica em evidências produzidas por estudos com alto rigor metodológico ser relativamente recente (cerca dos últimos 20 anos), as discussões acerca da efetividade em psicoterapia já ocorriam em meados dos anos 1950. Em uma publicação polêmica, Eysenck (1952), após revisar os estudos 
empíricos de psicoterapia de sua época, concluiu que as intervenções psicoterápicas utilizadas nas produções não eram mais efetivas para a evolução do quadro do paciente do que a simples passagem do tempo. Ainda segundo este autor, as psicoterapias utilizadas falharam quanto à recuperação dos pacientes, já que aproximadamente dois terços dos pacientes avaliados teriam uma evolução significativa após dois anos do início da doença, independentemente de serem tratados por meio de psicoterapia (Barlow, Boswell, \& Thompson-Hollands, 2013).

Eysenck (1952) concluiu que os dados de sua revisão não comprovavam que as psicoterapias praticadas na época facilitavam a recuperação dos pacientes neuróticos e que esta constatação era, notavelmente, estável de uma investigação para outra, independentemente do tipo de paciente tratado, do nível de recuperação empregado ou do método de terapia utilizada. Apesar dos achados e conclusões polêmicas do autor, vale salientar que ele compreendia que os dados mencionados não necessariamente refutavam a possibilidade de eficácia terapêutica, visto as dificuldades de comparação existentes, devido ao pouco acordo conceitual dos psiquiatras e psicoterapeutas da época (Barlow et al., 2013).

A publicação de Eysenck (1952) causou um considerável desconforto na comunidade de psicoterapeutas contemporâneos a ele, sendo que muitos tiveram reações contrárias aos apontamentos do autor, mesmo com a dificuldade de refutá-los com as evidências que haviam disponíveis, dado o precário estado desta ciência na década de 1960. Nos anos subsequentes à publicação do referido texto, diversos debates ocorreram na literatura, sendo que bons exemplos desses acalorados diálogos podem ser observados nas publicações de Rosenzweig (1954) e Strupp (1963, 1964), além das respostas de Eysenck (1955, 1964) (Barlow et al., 2013).

Rosenzweig (1954) rotulou o estudo de Eysenck (1952) como tendencioso, criticando veementemente a conceituação de neurose utilizada no estudo, as diferenciações do autor acerca das psicoterapias psicanalíticas, ecléticas e clínicas gerais, além da dificuldade de se avaliar a recuperação de pacientes cujos métodos de tratamento se diferem entre si. Tendo como base essas críticas, Rosenzweig (1954) concluiu que a única dedução segura com base nos dados que eram existentes é que, tendo em conta a diversidade de métodos e padrões no campo da psicoterapia, afirma- ções e generalizações quanto à eficácia dos tratamentos deveriam ser evitadas. Em sua resposta, Eysenck (1955), dentre outros apontamentos, salientou que, ao se perceber que os dados discutidos, pobres como são, compunham todas as evidências disponíveis a respeito de um método de psicoterapia praticado há mais de 50 anos em centenas de milhares de pacientes, eles deveriam servir como estímulos para garantir o início de estudos experimentais devidamente planejados, rigidamente controlados, e cuidadosamente analisados nesta área.

Strupp (1963; 1964), dentre suas críticas, reagiu aos dados de Eysenck (1952), expondo que haviam observações clínicas que documentavam os benefícios da relação com um profissional, quando pacientes estão preocupados com dificuldades que vivenciam e buscam auxílio. Segundo o crítico, argumentar o contrário do exposto seria, simplesmente, fechar os olhos para os fatos, já que todos sabiam que a psicoterapia era efetiva. Tal afirmação fora intensamente criticada por Eysenck (1964), que respondeu, sarcasticamente, que houve um momento em que todos sabiam que a Terra era plana (Barlow et al., 2013). Além desses debates, muitos psicoterapeutas da época relataram que as pesquisas em psicoterapia pouco contribuíam para a prática. Dentre eles, Carl Rogers, um dos mais importantes teóricos de psicoterapia do período, também observou que a pesquisa clínica não exercia nenhuma influência sobre a sua prática, podendo, portanto, ser abandonada completamente (Barlow et al., 2013; Bergin, \& Strupp, 1972).

Apesar dos dois tipos de reações que produziu, negar as conclusões dos dados ou questionar a aplicação da pesquisa clínica à prática profissional, o principal legado do polêmico estudo de Eysenck (1952) foi estimular a produção de pesquisas empíricas que demonstrassem os resultados das psicoterapias praticadas nas décadas de 1950 e 1960. Com isso, alguns estudos de revisão foram desenvolvidos nos anos subsequentes (por exemplo, Luborsky, Singer, \& Luborsky, 1975; Smith, \& Glass, 1977).

Na revisão de Luborsky et al. (1975), foram analisadas 105 pesquisas que confrontaram os efeitos de diferentes metodologias de psicoterapia, sendo elas psicanálise, terapia centrada no paciente, psicodrama e terapia comportamental, juntamente com comparações com grupos-controle e tratamentos farmacológicos. Como principal conclusão, os autores demonstraram que todos os pacientes tiveram benefícios ao 
final do processo terapêutico, independentemente da técnica utilizada.

De forma semelhante, o estudo de Smith e Glass (1977) forneceu evidências de que, em média, os pacientes tratados por meio de psicoterapia tinham probabilidade de melhora de $75 \%$ maior do que pacientes não tratados. Tal resultado foi produto de uma revisão de cerca de 400 estudos compostos por diferentes modalidades psicoterapêuticas. Vale salientar que não foram observadas diferenças na eficácia entre os tipos de psicoterapia analisados, sejam terapias comportamentais (Dessensibilização sistemática e Modificação de comportamento) ou terapias não comportamentais (Rogeriana, Psicodinâmica, Racional-emotiva, Análise transacional etc.).

Como visto, os estudos de revisão indicavam que a psicoterapia era altamente efetiva e que não existiam diferenças significativas nas diferentes modalidades de tratamento. Tais dados atribuíram a efetividade das psicoterapias às características presentes em diversificados processos psicoterapêuticos. Estas particularidades ficaram conhecidas como "Fatores Comuns" e incluíam propriedades do terapeuta (por exemplo, acolhimento, empatia, aceitação), do paciente (por exemplo, expectativas, busca pela melhora) e da relação terapêutica (por exemplo, transferência), considerada uma das principais variáveis do sucesso do tratamento (Barlow et al., 2013; DeRubeis, Brotman, \& Gibbons, 2005; Luborsky et al., 1975).

Alguns pesquisadores contemporâneos, contudo, não aceitaram os fatores comuns como principais responsáveis pelas mudanças no processo terapêutico, atribuindo aos fatores específicos de cada modalidade psicoterapêutica o sucesso das intervenções psicológicas. Para eles, a partir de uma queixa clínica, há uma explicação teórica acerca da gênese do problema e, por consequência, mecanismos de mudança presente nesta modalidade teórica. Tais mecanismos de mudança indicam, então, o procedimento psicoterapêutico a ser utilizado e o resultado da intervenção deveria ser conferido à terapêutica utilizada, ou seja, a um fator específico do processo de intervenção (Barlow et al., 2013; Beutler, 1979; Kazdin, \& Bass, 1989).

As discussões e conflitos acerca dos atributos responsáveis pelos resultados das psicoterapias se estenderam pelos anos que se seguiram, resultando em alguns movimentos da American Psychological Association (APA), que se iniciaram na década de 1990. Tais organizações ficaram conhecidas como forças-tarefa, sendo que a primeira fora realizada no ano de 1993 pela Divisão 12 da APA, que correspondia à área de Psicologia Clínica, com o intuito de comprovar o papel dos fatores específicos no sucesso dos processos psicoterapêuticos.

Composta por psicólogos de diferentes abordagens teóricas, com experiência em clínica e em pesquisa, a força-tarefa da Divisão 12 teve como objetivos: definir e identificar tratamentos empiricamente validados (TEV); propor diretrizes de formação para futuros terapeutas e de treinamento contínuo para os profissionais em exercício; disseminar os TEV para os planos de saúde; e informar a população sobre a efetividade da psicoterapia (Chambless, 1993; Chambless et al., 1998).

A busca por TEV foi realizada por meio de revisões em periódicos que continham publicações de pesquisas empíricas em psicoterapias, sendo que apenas em 1995 o relatório da força-tarefa da Divisão 12 da APA foi publicado. O resultado continha $18 \mathrm{TEV}$ e sete tratamentos provavelmente eficazes, sendo que as intervenções que não haviam sido incluídas nessas duas categorias deveriam ser consideradas, apenas, como experimentais (Chambless et al., 1998).

Segundo o relatório, para um tratamento ser considerado como "empiricamente validado", deveria existir dois ou mais ensaios clínicos randomizados conduzidos por diferentes pesquisadores (que demonstrassem que aquele tratamento era superior a placebo ou a outras intervenções psicoterápicas ou que era equivalente a um tratamento já estabelecido em estudos estatisticamente significantes) e nove ou mais pesquisas com delineamento experimental de caso único com alta qualidade metodológica (que demonstrassem que aquele tratamento era superior a placebo ou a outros tratamentos já estabelecidos). Para um tratamento ser considerado como "provavelmente eficaz", o relatório apresenta que deveriam existir dois estudos de grupo (que demonstrassem que aquele tratamento era superior a um grupo sem tratamento) ou três ou mais pesquisas com delineamento experimental de caso único com boa qualidade metodológica (que demonstrassem que aquele tratamento era superior a placebo ou a outras intervenções). Vale salientar que um tratamento também era listado como "provavelmente eficaz" quando atendia a todos os critérios para ser considerado como empiricamente validado, mas os estudos que o sustentavam haviam sido realizados pelo mesmo grupo de pesquisadores (Task Force on Promotion and Dissemination of Psychological Procedures, 1995). 
Algumas atualizações do relatório inicial ocorreram nos anos que se seguiram. A primeira substituiu a expressão Tratamentos empiricamente validados (TEV) para Tratamentos empiricamente sustentados (TES). Outras alterações foram: a organização de tratamentos por transtornos específicos e a inserção de novos tratamentos e algumas alterações na lista anterior, sendo que foram considerados apenas os tratamentos cujos procedimentos estavam padronizados em manuais de intervenção, visto que isso poderia contribuir para um melhor delineamento de pesquisa e interpretação dos resultados (Chambless et al., 1996).

Outra atualização ocorreu em 1998, na qual uma nova lista de TES foi publicada, relacionando 16 tratamentos empiricamente sustentados e 55 tratamentos provavelmente eficazes, além da exclusão de dois procedimentos listados anteriormente, já que não se referiam a problemas específicos e poderiam ser empregados em uma diversidade de situações (Chambless et al., 1998). Além disso, houve uma publicação de uma lista atualizada dos manuais com os procedimentos dos TES (Woody, \& Sanderson, 1998).

Apesar do avanço e das conquistas da Força Tarefa da Divisão 12, o movimento recebeu inúmeras críticas de profissionais e pesquisadores da época. Os julgamentos relacionavam-se especificamente em três áreas: diagnóstico, utilização de manuais e metodologia utilizada nas pesquisas (Addis, Wade \& Hatgis, 1999; Bohart, O'Hara, \& Leitner, 1998; Chambless, \& Ollendick, 2001; Reed, Kihlstrom, \& Messer, 2006; Westen, Stirman, \& DeRubeis, 2006).

Ainclusão somente de pesquisas cujos participantes preenchiam os critérios diagnósticos para alguma psicopatologia descrita no Eixo I do Manual Diagnóstico e Estatístico de Transtornos Mentais - DSM (American Psychiatric Association, 2002) foi criticada, visto que os diagnósticos não capturavam as particularidades que os clientes levam para o consultório e nem os contextos em que os problemas surgem e se mantêm. Além disso, rotular um grupo de indivíduos apenas por compartilharem algumas características não os torna semelhantes, ao contrário, cada paciente deveria ser visto como único e, portanto, receber um tratamento totalmente individualizado (Bohart et al., 1998; Reed et al., 2006).

Com relação a utilização dos manuais de tratamento, os profissionais e pesquisadores reagiram negativamente devido ao fato de que os manuais, por apresentam protocolos padronizados de interven- ção que devem ser seguidos fielmente pelo terapeuta frente a problemas clínicos específicos, atrapalhariam o desenvolvimento de uma boa relação terapêutica, ignoraria as diferenças individuais de cada cliente, não atenderia às necessidades de clientes com múltiplos problemas e/ou diagnósticos, ameaçaria a independência, espontaneidade e criatividade do clínico etc. (Addis, Wade, \& Hatgis, 1999).

Quanto ao método realizado para a avaliação dos tratamentos, a força-tarefa recebeu três grupos de críticas por considerar os Ensaios Clínicos Randomizados (ECR) como o padrão-ouro para a produção de evidências. Os estudos de grupo, basicamente quantitativos, seriam inadequados para o campo da psicoterapia, sendo a pesquisa qualitativa a mais conveniente; as abordagens comportamentais e cognitivas teriam levado vantagem na avaliação, pois foram mais pesquisadas com esse tipo de método; e o fato de um tratamento ser eficaz em condições controladas (validade interna), não garantiria que ele seria efetivo em ambiente real (validade externa) (Chambless, \& Ollendick, 2001; Westen et al., 2006).

Concomitantemente às críticas direcionadas ao trabalho da Divisão 12, outras divisões da APA realizaram suas forças-tarefa, cujo intuito fora complementar as possíveis falhas na avaliação das intervenções psicoterápicas. Em 1999, por exemplo, a Divisão 29 (Psicoterapia) formou sua força-tarefa com o objetivo de identificar, operacionalizar e disseminar dados acerca de relações terapêuticas empiricamente sustentadas. Assim, uma revisão de pesquisas quantitativas e qualitativas deu origem a uma listagem de elementos da relação terapêutica e de características do paciente que contribuiriam para o resultado do processo terapêutico (Norcross, 2002; Wampold, 2001).

A tensão entre as forças-tarefa das diferentes divisões da APA intensificou o debate sobre o que deveria ser empiricamente validado na pesquisa em psicoterapia - fatores comuns ou fatores específicos. Em meio ao embate entre os defensores dos fatores comuns e os defensores dos fatores específicos, uma força-tarefa foi constituída em 2002 pela Society for Psychotherapy Research (SPR) em conjunto com a Divisão 12 da APA, cujo objetivo era a de que todos os fatores envolvidos no processo psicoterápico estariam relacionados ao resultado e, portanto, deveriam ser considerados. Para isso, diversos estudos foram revisados, levando em conta quatro grupos de problemas clínicos: transtornos disfóricos, transtornos de 
ansiedade, transtornos de personalidade e transtornos relacionados ao uso de substâncias. Essa revisão deu origem à formulação de princípios de mudança terapêutica (PMT), que poderiam ser aplicados em qualquer modalidade de psicoterapia, uma vez que englobavam variáveis relacionadas ao terapeuta, paciente, relação terapêutica e técnicas extraídas da literatura empírica que foram apresentadas sem ligação com qualquer teoria (Beutler, \& Johannsen, 2006; Castonguay, \& Beutler, 2006a, 2006b).

Em meio aos debates entre os defensores dos fatores comuns e dos fatores específicos, e com o objetivo de aproximação entre ciência e prática, a APA iniciou, em 2005, uma força-tarefa composta por psicólogos de diferentes abordagens, com o objetivo de desenvolver uma concepção de prática baseada em evidências que fosse representativa das mais diversas perspectivas teóricas, metodológicas, conceituais e práticas. Deste modo, a APA definiu o conceito de Prática Baseada em Evidências em Psicologia (PBEP) como um processo de tomada de decisão clínica, que ocorre por meio da integração da melhor evidência disponível com a perícia clínica do psicoterapeuta, no contexto das características, cultura e preferências do paciente, sendo que os três componentes da definição possuem o mesmo grau de importância na decisão clínica (American Psychological Association, 2006).

O primeiro conceito da definição - melhor pesquisa disponível - refere-se às evidências empíricas que demonstrem os procedimentos psicoterapêuticos e os resultados positivos, a depender do objetivo em questão. Tais evidências podem ser fruto de diversos tipos de pesquisa: eficácia, efetividade, custo-benefício, epidemiológica etc., envolvendo diferentes metodologias: ECR, delineamentos de caso único, estudos de caso etc. (American Psychological Association, 2006). Além do exposto, vale salientar que o conceito abrange a existência de dados empíricos com diferentes níveis de qualidade, ficando a cargo do terapeuta a seleção das pesquisas mais relevantes a partir do grau de compatibilidade das evidências disponíveis com o caso em questão e do grau de confiabilidade dessas evidências, determinado pela quantidade de pesquisas e pela qualidade metodológica das mesmas (Spencer, Detrich, \& Slocum, 2012).

Com relação à perícia clínica, a APA a compreende como o repertório especializado do psicoterapeuta, constituído por sua formação acadêmica, supervisão, experiência clínica e estudo da literatura.
Esse repertório inclui diversas competências e, em especial, a obtenção e aplicação das melhores evidências disponíveis para cada caso particular. Dessa forma, deve haver interação constante entre o julgamento profissional e os dados existentes (American Psychological Association, 2006).

O terceiro componente da definição de PBEP - contexto das características, cultura e preferências do paciente - se caracteriza pela promoção da participação ativa dos clientes nas tomadas de decisão sobre sua própria saúde e bem-estar, uma vez que intervenções costumam ser mais bem-sucedidas quando levam em consideração as particularidades do indivíduo (American Psychological Association, 2006). A integração das preferências do paciente com os outros componentes da definição de PBEP é uma tarefa complexa. Em vista disso, é responsabilidade do terapeuta descrever os procedimentos a serem empregados, explicar sobre seus possíveis benefícios, riscos e inconvenientes e advertir sobre intervenções alternativas (Spencer et al., 2012).

Na prática clínica, o modelo de PBEP pode ser operacionalizado em cinco passos: levantamento de questões clínicas; busca pelas melhores evidências empíricas, principalmente em bancos de dados que são voltados para profissionais atuantes e não para pesquisadores e que, por isso, reúnem apenas revisões sistemáticas da literatura empírica; apreciação crítica das evidências encontradas; intervenção, baseada nas evidências, no repertório do terapeuta e nas características do cliente; avaliação dos resultados da intervenção (Frueh, Ford, Elhai, \& Grubaugh, 2012; Walker, \& London, 2007).

Como dito, o conceito de PBEP está no centro das atenções do cenário internacional da Psicologia e constantes esforços têm sido feitos nessa direção por pesquisadores e profissionais, com o intuito de preencher a lacuna entre ciência e prática ainda existente na Psicologia Clínica. Todavia, embora a PBEP venha sendo amplamente defendida entre pesquisadores da Psicologia, ela apresenta como limitação o fato das publicações focarem-se quase que exclusivamente no modelo conceitual de PBEP, sem atenção ao ensino das habilidades necessárias para sua aplicação. O contrário ocorre na medicina, visto que o desenvolvimento de habilidades práticas ocupa $60 \%$ ou mais das publicações (Walker, \& London, 2007). Vale salientar que o modelo da Medicina Baseada em Evidências teve, e ainda tem, grande participação na conceitua- 
ção e no molde de aplicação da PBEP, mesmo tendo premissas teóricas e metodológicas questionáveis para utilização em psicoterapias.

\section{Prática baseada em evidências em Psicologia e idosos}

Em revisão recente, Monteleone e Witter (2014) analisaram os estudos que correlacionam o conceito de PBEP com a população idosa, na base de dados da PsycINFO. Apesar da escassez de produções (72 estudos), dentre os principais resultados destaca-se a ascensão de estudos nos últimos três anos, sendo que as entidades promotoras, bem como os periódicos de publicação das pesquisas concentram-se, em sua maioria, nos Estados Unidos da América. Com relação ao tipo de pesquisa, $62 \%$ das produções eram descritivas e $48 \%$ dos estudos eram experimentais. Vale ressaltar que grande parte dos estudos experimentais comparava modalidades de psicoterapias com fármacos, em intervenções em demências e outras doenças comuns à população idosa.

Psychology and Aging é um periódico da APA, indexado na PsycINFO, que publica artigos originais sobre o desenvolvimento de adultos e envelhecimento. Tais produções incluem relatos de pesquisas que podem ser dos seguintes tipos: aplicadas, bio-comportamentais, clínicos, educacionais, experimentais, metodológicos ou psicossociais. A página oficial do periódico pode ser acessada em http://www.apa.org/pubs/journals/pag/index.aspx.

A partir da publicação das políticas de compromisso de prática com base em evidências de pesquisas pela APA, alguns estudos foram desenvolvidos com intuito de reunir e estabelecer as melhores informações científicas sobre determinado tema. Especificamente com a população idosa, o periódico Psychology and Aging propôs, em 2007, uma seção especial que reuniu quatro revisões sistemáticas que, somadas a um estudo anterior, visavam situar os tratamentos mais efetivos para cinco queixas clínicas: depressão, insônia, ansiedade, sobrecarga no cuidador e distúrbios comportamentais na demência (Scogin, 2007).

Por meio de um manual de codificação desenvolvido por Weisz e Hawley $(2001)^{1}$, as revisões foram desenvolvidas por equipes de peritos em cada queixa clínica. O processo foi desenvolvido em cinco fases para cada revisão, sendo que todo o desenvolvimento dos estudos foi monitorado e supervisionado pelas autoridades da Sociedade de Psicologia Clínica da APA (Yon, \& Scogin, 2007).

Na primeira fase, o presidente da comissão, Forrest Scogin, que já havia realizado uma revisão sistemática sobre depressão (Scogin, Welsh, Hanson, Stump, \& Coates, 2005), contatou os especialistas em cada área para revisar os estudos de intervenção sobre: transtornos de ansiedade, distúrbios comportamentais na demência, angústia no cuidador e distúrbios do sono. Estes profissionais reuniram equipes de revisores e treinaram os membros para se familiarizarem com o manual de codificação e com o processo de revisão (Yon, \& Scogin, 2007).

A segunda fase consistiu em uma revisão da literatura para identificar artigos possivelmente relevantes. Tais estudos deveriam ter passado por um processo de revisão por pares, sendo que cada equipe revisou toda a literatura em sua área publicada até dezembro de 2005. Para identificar potenciais estudos, os revisores de cada equipe usaram como bases para a busca os eletrônicos: PsycINFO, Academic Search Elite, PubMed, Medline além da Cochrane Collaboration.

A equipe de revisão sobre ansiedade geriátrica identificou 77 estudos (Ayers, Sorrell, Thorp, \& Wetherell, 2007). Cinquenta e sete pesquisas foram identificados pela equipe de revisão de distúrbios comportamentais em literatura de demência (Logsdon, McCurry, \& Teri, 2007). A equipe de revisão de sobrecarga do cuidador considerou mais de 350 artigos (Gallagher-Thompson \& Coon, 2007). A revisão da literatura sobre insônia identificou mais de 100 estudos (McCurry, Logsdon, Teri, \& Vitiello, 2007).

No terceiro passo, após a literatura ser identificada, os revisores classificaram cada artigo com base nos critérios delineados no manual de codificação. Vários requisitos fundamentais foram exigidos para incluir as pesquisas nos critérios para seleção: tanto entre os grupos (com atribuição aleatória) e dentro do grupo, os estudos eram aceitáveis com um mínimo de 30 participantes, na faixa etária apropriada (com idades de 60 anos ou mais, com exceção da revisão sobre cuidadores); pelo menos dois estudos deveriam ter recebido o tratamento em questão; o tratamento

${ }^{1}$ Weisz, J. R., \& Hawley, K. M. (2001). Procedural and coding manual for identification of evidence-based treatments. University of California, LA. [Unpublished]. 
deveria também, ter sido aplicado a indivíduos com o mesmo problema-alvo e descrito minuciosamente em manuais de aplicação.

Para ser classificado como uma contribuição para uma prática com base em evidências, os resultados deveriam indicar que o tratamento era melhor do que uma condição de controle ou comparável a um tratamento baseado em evidências já estabelecido. O manual de codificação estipula que um tratamento é considerado melhor do que uma condição de controle quando pelo menos $50 \%$ das medidas de resultados que avaliam o problema alvo exibir significância aceitável na área $(\alpha<0,05)$, entre os efeitos do tratamento em grupo.

Após a realização dos critérios de exclusão, a equipe de revisão da literatura sobre ansiedade descartou 60 estudos; 49 pesquisas foram excluídas da análise dos distúrbios de comportamento na literatura de demência; a equipe de revisão que estudou a sobrecarga do cuidador eliminou 299 estudos; 89 pesquisas foram eliminadas pela equipe de revisão da literatura sobre insônia geriátrica. Com as exclusões dos artigos que não preencheram os critérios necessários, a amostra que compôs cada revisão pode ser observada na Tabela.

Na quarta fase, as decisões de codificação feitas pelos revisores independentes foram comparadas. Quando ocorriam discrepâncias, os dados eram discutidos pela equipe de revisão, que produziam as decisões finais por meio de um consenso da maioria (Yon \& Scogin, 2007). A quinta e última fase do processo de revisão consistiu na divulgação dos resultados entre as equipes para que, a partir de uma análise coletiva, todas as dúvidas fossem sanadas, a fim de que as últimas decisões pudessem ser tomadas. Uma vez analisada por outros membros, cada equipe de revisão realizou os ajustes necessários para que, na sequência, os manuscritos fossem preparados e submetidos ao processo de revisão por pares da revista, antes da publicação.

Seguindo pela seção especial do periódico, a primeira revisão refere-se ao estudo de Ayers et al. (2007), cujo foco foi tratamentos psicológicos para Transtorno de Ansiedade Generalizada (TAG) ou variações da ansiedade associada a outros diagnósticos. Os estudos foram inclusos conforme estabelecido para a seção especial, sendo que, particularmente para esta revisão, as pesquisas deveriam ser randomizadas e controladas, em que uma intervenção fosse comparada com uma lista de espera, um tratamento usual, uma intervenção alternativa ou uma condição de placebo, além de ter pelo menos uma medida de resultado objetiva. Como resultados, a revisão classificou, por meio dos 17 estudos analisados, quatro tratamentos baseados em evidência, sendo eles: terapia cognitivo-comportamental (nove estudos); treinamento de relaxamento (quatro estudos); terapia cognitiva (um estudo); e terapia de suporte (três estudos).

Na revisão de McCurry et al. (2007), cujo objetivo fora verificar tratamentos baseados em evidências para insônia em idosos, foi identificada duas modalidades de intervenção, por meio da análise de 20 estudos. A primeira refere-se à terapia de restrição de sono e a segunda à terapia cognitivo-comportamental com multicomponentes.

O terceiro estudo da sessão especial focou na constatação de tratamentos baseados em evidência para distúrbios comportamentais na demência. Para isso, foram utilizadas 14 pesquisas, em que duas intervenções atenderam aos critérios: "Redução Progressiva do Limiar de Estresse" e "Terapia para Resolução de Problemas" (Logsdon et al., 2007).

Com relação à revisão de Gallagher-Thompson e Coon (2007), os resultados sugeriram que três méto-

Tabela

Perfil dos estudos analisados.

\begin{tabular}{lccc}
\hline Revisão e equipe & $\begin{array}{c}\text { Estudos } \\
\text { identificados }\end{array}$ & $\begin{array}{c}\text { Estudos } \\
\text { eliminados }\end{array}$ & $\begin{array}{c}\text { Estudos } \\
\text { analisados }\end{array}$ \\
\hline Ansiedade (Ayers et al., 2007) & 77 & 60 & 17 \\
$\begin{array}{l}\text { Distúrbios Comportamentais na Demência } \\
\text { (Logsdon et al., 2007) }\end{array}$ & 57 & 43 & 14 \\
Sobrecarga no cuidador (Gallagher-Thompson, \& Coon, 2007) & 359 & 299 & 60 \\
Insônia (McCurry et al., 2007) & 109 & 89 & 20 \\
\hline
\end{tabular}


dos de intervenção atenderam aos critérios de tratamentos baseados em evidência para sobrecarga no cuidador. A partir da análise de 60 estudos, pôde-se verificar a efetividade de Psicoeducação para Construção de Habilidades; Aconselhamento Psicoterápico; e Intervenção com Multicomponentes.

Pôde-se perceber uma uniformidade no método de revisão de todos os estudos, bem como uma padronização quanto à forma de apresentação e discussão dos resultados na sessão especial. Os procedimentos utilizados para o desenvolvimento das pesquisas consistiram em um refinamento dos métodos empregados por Scogin et al. (2005), em uma revisão acerca da efetividade de tratamentos psicológicos para depressão, por meio de 35 estudos analisados. Apesar de não fazer parte diretamente da seção especial aqui discutida, os dados apresentam relevância ímpar, visto a prevalência deste transtorno na população idosa.

Os principais resultados sugerem que Terapias Comportamentais e Terapias Cognitivo-Comportamentais apresentaram melhora significativa, além dos ganhos serem mantidos por maior período do que terapias psicodinâmicas breves. Somadas a essas terapêuticas, foi observada efetividade em processo com Terapias por Insight; Terapia de Resolução de Problemas; Terapia Cognitiva; e Biblioterapia Cognitiva (Scogin, et al. 2005).

Ao se analisar criticamente todas as revisões apresentadas, algumas considerações se fazem necessárias para o presente estudo. A primeira refere-se aos critérios de seleção da amostra, já que houve um grande descarte em todos os estudos, visto que mais de $50 \%$ das produções foram excluídas por não se adequarem aos critérios de seleção. A inclusão de estudos cujas amostras foram constituídas apenas de grupos com o mínimo de 30 participantes teve influência significativa no número de pesquisas analisadas, atendendo a critérios provenientes das revisões médicas, além de não considerar especificidades de áreas da Psicologia que delineiam suas experimentações por meio de estudos com casos-único.

Outra consideração se refere à utilização de manuais para a padronização das intervenções. Não são todas as áreas da Psicologia que utilizam protocolos padronizados para atendimento clínico, visto a pluralidade de conceitos teóricos que inviabilizam a utilização de procedimentos uniformizados, sem a adequação para cada cliente. Ainda sobre os manuais, os estudos de revisão pouco apresentam dados sobre o processo terapêutico, limitando-se na maioria dos casos, à referência do protocolo.

Em última análise, observou-se que, apesar dos artigos introdutórios da seção especial descreverem brevemente o processo de codificação, as revisões não discutiram as estatísticas utilizadas neste processo, além de pouco apresentar os valores que indicam evidência favorável para as terapêuticas analisadas. Outro fator observado foi o fato da não utilização de meta-análises como forma de avaliação do nível de evidência de cada estudo.

A inserção de estudos com sujeito-único, o desenvolvimento de medidas de resultado específicas para a Psicologia e o desenvolvimento de estudos do processo dos tratamentos podem ser alternativas para a ampliação e solidificação do conhecimento produzido pelas revisões apresentadas. A utilização de pesquisas com delineamento experimental de caso-único pode sugerir as diferenciações no processo de mudança para cada sujeito e para cada procedimento terapêutico; o desenvolvimento de medidas específicas para a área tende a padronizar a análise dos resultados e, somadas aos instrumentos clássicos, podem ampliar o diálogo com as outras ciências; juntamente com a análise sobre o processo terapêutico pode sugerir possíveis variáveis independentes para estudos de aplicação, posteriores. Aliada às tais premissas, a aplicação de métodos estatísticos de alta qualidade pode ser cabível para a obtenção dos tratamentos com melhores resultados a partir de sua experimentação em pesquisa.

Estudos com sujeito-único têm sido utilizados e defendidos por diversos pesquisadores. A principal especificidade deste desenho experimental refere-se ao participante sendo seu próprio controle, em momentos diferenciados no estudo. Deste modo, as medidas são feitas de forma sistemática ao longo de todo o processo e não apenas em momentos pré e pós intervenção, como tradicionalmente são feitas (Ferster, \& Skinner, 1957; Hersen, \& Barlow, 1972; Kazdin, 1981; Oshiro, 2011; Oshiro, Kanter, \& Meyer, 2012; Sidman, 1960).

Neste contexto, diversas variações são utilizadas para delinear as condições dos experimentos com caso-único (por exemplo, Delineamento de Reversão; Delineamento de linha de Base Múltipla; Delineamento de Mudança de Critério; Delineamento de Sonda; Delineamento de Retirada etc.). Os delineamentos de caso-único têm como principal carac- 
terística, analisar os participantes individualmente, tanto nas decisões relativas ao desenho experimental, quanto na avaliação dos dados. Diferentemente dos estudos com grupos, um mesmo participante é submetido a todas as condições do experimento e as observações e medidas são realizadas de forma contínua no decorrer do processo (Sampaio et al., 2008).

Em publicação clássica sobre o tema, Kazdin (1981) discutiu a possibilidade de concepção e condução de estudos com sujeito-único, de forma a maximizar a probabilidade de obtenção de conclusões válidas. Para este autor, para que a validade interna dos resultados não seja ameaçada, os estudos de sujeito-único devem conter avaliação contínua em medidas objetivas; a queixa-alvo deve apresentar estabilidade nas medidas antes da condição experimental; e os efeitos da intervenção devem ser imediatos e significativos. Além destes critérios, o experimento deve ser realizado nas mesmas condições em múltiplos casos, apresentando efeitos semelhantes.

Em consonância com Kazdin, a APA (s.d.) apresenta diretrizes para os estudos de sujeito único, para que os resultados possam ser considerados como baseados em evidência. Para a instituição, o estudo deve conter o mínimo de duas medidas de resultados padronizadas, bem como uma medida do processo avaliada em três condições do estudo e os resultados devem ser apresentados utilizando-se diferença média padronizada e significação clínica, em ao menos três participantes diferentes.

Por meio deste desenho experimental, investigações sobre aspectos de uma modalidade de psicoterapia que resultem em mudança podem ser realizadas, de modo a observar parcelas do resultado global e elucidar aspectos da relação terapêutica que propiciaram a mudança observada. Neste sentido, a variação intra-sujeito presente nos estudos de sujeito único é fundamental para pesquisas em psicoterapia (Oshiro, 2011).

Outra possível contribuição para as revisões já discutidas é a utilização de técnicas estatísticas para a combinação dos diferentes resultados encontrados. A utilização de meta-análises integra e refina os efeitos de diversas intervenções, de modo a sugerirem quais procedimentos produziram maior significância estatística. Na Psicologia, as revisões Sistemáticas que utilizam meta-análises são pouco frequentes, sendo que grande parte dos estudos existentes foram conduzidos por equipes da Colaboração Cochrane (Cochrane
Colaboration: http://www.cochrane.org), instituição esta que é mundialmente conhecida por produzir revisões sistemáticas, com ou sem meta-análises, nas mais diversas modalidades de intervenção em saúde (Atallah, \& Puga, 2011; Melnik, \& Atallah, 2011).

A inserção de métodos quantitativos que combinem e analisem os resultados das intervenções com idosos pode sugerir possíveis intervenções que se destaquem do quórum analisado, podendo possibilitar a identificação de variáveis independentes primordiais para estudos de aplicação subsequentes, com alto rigor metodológico. Além dos ganhos acadêmicos, tal processo pode embasar intervenções clínicas em ambiente profissional, visto o aumento da demanda desta população para os serviços de psicoterapia.

\section{Considerações finais}

O presente artigo teve por objetivo apresentar o desenvolvimento histórico do movimento de Prática Baseada em Evidências em Psicologia, bem como a integração deste modelo com a atuação psicoterápica junto à população idosa. Deste modo, pôde-se perceber que a construção histórica deste movimento sofreu diversas influências, além de ter sido estabelecida em contextos conflituosos, o que, aparentemente, produz controvérsias ainda nos dias atuais. Neste contexto, a busca por evidências de resultado de intervenções psicoterápicas para a população idosa ocorre de forma ainda deficitária, com pouquíssimos estudos e revisões com critérios discutíveis e não claramente definidos.

A inserção de outras variáveis metodológicas em estudos que abordem os resultados de intervenções psicoterápicas com a população idosa parece ser uma alternativa proeminente para o desenvolvimento de modalidades psicoterapêuticas com esta população. De modo semelhante, a adoção de novos critérios para os estudos de revisão sistemática que visem mensurar o efeito destas intervenções junto aos idosos pode fornecer novos indicativos de modalidades psicoterapêuticas empiricamente sustentadas para determinadas queixas. Sobre estes critérios, destaca-se aqui a inserção de estudos com delineamentos experimentais de caso-único ou com outros arranjos, não limitando a amostra a apenas ensaios clínicos randomizados, e a utilização de métricas estatísticas em meta-análises, quando possível, como forma padronizada de mensuração do efeito das variadas modalidades de intervenção. Entretanto, se faz neces- 
sário que estes critérios considerem elementos culturais, sociais e econômicos, visto que a velhice se configura em uma diversidade de processos em que esses fatores se inter-relacionam.

Obviamente, apesar de apresentar os principais estudos existentes sobre a temática aqui analisada, este artigo limita-se a uma discussão teórica acerca dos temas abordados. Dessa forma, o desenvolvimento futuro, sobretudo nacionalmente, da Psicologia como profissão e prática científica depende do empenho contínuo dos principais envolvidos, em vistas a inserção da Psicologia nacional neste desenvolvimento, além da real aproximação da prática profissional à ciência psicológica.

\section{Referências}

Addis, M. E., Wade, W. A., \& Hatgis, C. (1999). Barriers to dissemination of evidence-based practices: addressing practitioners' concerns about manual-based psychotherapies. Clinical Psychology: Science and Practice, 6(4), 430-441. https://doi.org/10.1093/clipsy.6.4.430

American Psychological Association. (s.d.). Evidence-based case study. Recuperado de http://www.apa.org/pubs/journals/pst/evidence-based-case-study.aspx

American Psychological Association. (2006). Evidence-based practice in psychology: APA presidential task force on evidence-based practice. American Psychologist, 61(4), 271-285. https://doi.org/10.1037/0003-066X.61.4.271

American Psychiatric Association. (2002). Manual diagnóstico e estatístico de transtornos mentais (4a ed.; C. Dornelles, Trad.). Porto Alegre, RS: Artmed.

Atallah, A. N., \& Puga, M. E. S. (2011). A colaboração Cochrane e o seu papel como produtora das melhores evidências. In T. Mielnick, \&A. N. Atallah (Orgs), Psicologia baseada em evidências: provas científicas da efetividade da psicoterapia. São Paulo, SP: Grupo Gen.

Ayers, C. R., Sorrell, J. T., Thorp, S. R., \&Wetherell, J. L. (2007). Evidence-based psychological treatments for late-life anxiety. Psychology and Aging, 22(1), 8-17. https://doi.org/10.1037/0882-7974.22.1.8

Barlow, D. H., Boswell, J. F., \& Thompson-Hollands, J. (2013). Eysenck, Strupp, and 50 years of psychotherapy research: a personal perspective. Psychotherapy (Chic), 50(1), 77-87. https://doi.org/10.1037/a0031096

Bergin, A. E., \& Strupp, H. H. (1972). Changing frontiers in the science of psychotherapy. Chicago, Ill: Aldine Atherton.

Beutler, L. E. (1979). Toward specific psychological therapies for specific conditions. Journal of Consulting and Clinical Psychology, 47(5), 882-897.

Beutler, L. E., \& Johannsen, B. E. (2006). Principles of change. In J. C. Norcross, L. E. Beutler, \& R. F. Levant (Eds.), Evidence-based practices in mental health: debate and dialogue on the fundamental questions (pp. 226-234). Washington. DC: American Psychological Association.

Bohart, A. D., O'Hara, M., \& Leitner, L. M. (1998). Empirically violated treatments: disenfranchisement of humanistic andotherpsychotherapies. PsychotherapyResearch, 8(2),141-157.https://doi.org/10.1080/10503309812331332277

Castonguay, L. G., \& Beutler, L. E. (2006a). Principles of therapeutic change: a task force on participants, relationships, and techniques factors. Journal of Clinical Psychology, 62(6), 631-638. https://doi.org/10.1002/jclp.20256

Castonguay, L. G., \& Beutler, L. E. (Eds.). (2006b). Principles of therapeutic change that work. New York, NY: Oxford University Press.

Chambless, D. L. (1993). Task force on promotion and dissemination of psychological procedures: a report adopted by the Division 12 Board. Washington. DC: American Psychological Association.

Chambless, D. L., Baker, M., Baucom, D. H., Beutler L. E, Calhoun K. S., Crits-Christoph, P. et al. (1998). Update on empirically validated therapies, II. The Clinical Psychologist, 51, 3-16.

Chambless, D. L., \& Ollendick, T. (2001). Empirically supported psychological interventions: controversies and evidence. Annual Review of Psychology, 52, 685-716. https://doi.org/10.1146/annurev.psych.52.1.685

Chambless, D. L., Sanderson, W. C., Shoham, V., Johnson, S. B., Pope, K. S., Crits-Christoph, P. et al. (1996). An update on empirically validated therapies. The Clinical Psychologist, 49, 5-18. 
DeRubeis, R. J., Brotman, M. A., \& Gibbons, C. J. (2005). A conceptual and methodological analysis of the non-specific argument. Clinical Psychology: Science and Practice, 12, 174-183.

Eysenck, H. J. (1952). The effects of psychotherapy: an evaluation. Journal of Consulting Psychology, 16(5), 319-324.

Eysenck, H. J. (1955). The effects of psychotherapy: a reply. Psychotherapy, 50(1), 147-148.

Eysenck, H. J. (1964). The outcome problem in psychotherapy: a reply. Psychotherapy (Chic), 1(3), 97-100.

Ferster, C. B., \& Skinner, B. F. (1957). Schedules of reinforcement. New York, NY: Appleton-Century-Crofts.

Frueh, B. C., Ford, J. D., Elhai, J. D., \& Grubaugh, A. L. (2012). Evidence-based practice in adult mental health. In P. Sturmey, \& M. Hersen (Eds.), Handbook of evidence-based practice in clinical psychology, volume two: adult disorders (pp. 3-14). Hoboken, NJ: John Wiley \& Sons.

Gallagher-Thompson, D., \& Coon, D. W. (2007). Evidence-based psychological treatments for distress in family caregivers of older adults. Psychology and Aging, 22(1), 37-51. https:// doi.org/10.1037/0882-7974.22.1.37

Hersen, M., \& Barlow, D. H. (1976). Single case experimental designs: strategies for studying behavior change. Oxford: Pergamon.

Kazdim, A. E. (1981). Drawing valid inferences from case studies. Journal of Consulting and Clinical Psychology, 49(2), 183-192. https://doi.org/10.1037/0022-006X.49.2.183

Kazdin, A. E., \& Bass, D. (1989). Power to detect differences between alternative treatments in comparative psychotherapy outcome research. Journal of Consulting and Clinical Psychology, 57(1), 138-147. https://doi.org/10.1037/0022-006X.57.1.138

Logsdon, R. G., McCurry, S. M., \& Teri, L. (2007). Evidence-based psychological treatments for disruptive behaviors in individuals with dementia. Psychology and Aging, 22(1), 28-36.

Luborsky, L., Singer, B. H., \& Luborsky, L. (1975). Comparative studies of psychotherapies: Is it true that "everyone has won and all must have prizes"? Archives of General Psychiatry, 32(8), 995-1008.

McCurry, S. M., Logsdon, R. G., Teri, L., \& Vitiello, M. V. (2007). Evidence-based psychological treatments for insomnia in older adults. Psychology and Aging, 22(1), 18-27. https://doi.org/10.1037/0882-7974.22.1.18

Melnik, T., \& Atallah, A. N. (2011). Psicologia baseada em evidências: articulação entre a pesquisa e prática clínica. In T. Mielnick', \& A. N. Atallah (Orgs.), Psicologia baseada em evidências: provas científicas da efetividade da psicoterapia. São Paulo, SP: Grupo Gen.

Monteleone, T. V., \& Witter (2014). Prática baseada em evidências em psicologia com idosos: análise da produção científica. In Anais da $44^{a}$ Reunião Anual da Sociedade Brasileira de Psicologia (pp. 33-34). Ribeirão Preto, SP: Sociedade Brasileira de Psicologia.

Norcross, J. C. (Ed.). (2002). Psychotherapy relationships that work: therapist contributions and responsiveness to patient needs. New York, NY: Oxford University Press.

Oshiro, C. K. B. (2011). Delineamento experimental de caso único: a psicoterapia analítica funcional com dois clientes dificeis (Tese de Doutorado). Instituto de Psicologia, Universidade de São Paulo, São Paulo.

Oshiro, C. K. B., Kanter, J. \& Meyer S. B. (2012). A single-case experimental demonstration of functional analytic psychotherapy with two clients with severe interpersonal problems. International Journal of Behavioral Consultation and Therapy, 7(2-3), 111-116. https://doi.org/10.1037/h0100945

Reed, G. M., Kihlstrom, J. F., \& Messer, S. B. (2006). What qualifies as evidence of effective practice? In J. C. Norcross, L. E. Beutler, \& R. F. Levant (Eds.), Evidence-based practices in mental health: debate and dialogue on the fundamental questions (pp. 13-55). Washington, DC: American Psychological Association.

Rosenzweig, S. (1954). A transvaluation of psychotherapy: a reply to Hans Eysenck. Journal of Abnormal and Social Psychology, 49(2), 298-304.

Sampaio, A. A. S., Azevedo, F. H. B., Cardoso, L. R. D., Lima, C., Pereira, M. B. R. \& Andery, M. A. P. A. (2008). Uma introdução aos delineamentos experimentais de sujeito único. Interação em Psicologia, 12(1), 151-164. Recuperado de http://revistas.ufpr.br/psicologia/article/viewFile/9537/9218..

Scogin, F. (2007). Introduction to the special section on evidence-based psychological treatments for older adults. Psychology and Aging, 22(1), 1-3. https:// doi.org/10.1037/0882-7974.22.1.1 
Scogin, F., Welsh, D., Hanson, A., Stump, J., \& Coates, A. (2005). Evidence-based psychotherapies for depression in older adults. Clinical Psychology: Science and Practice, 12(3), 222-237. https://doi.org/10.1093/clipsy.bpi033

Sidman, M. (1960). Tactics of scientific research. New York, NY: Basic Books.

Smith, M. L., \& Glass, G. V. (1977). Meta-analysis of psychotherapy outcome studies. American Psychologist, 32(9), 752-760.

Spencer, T. D., Detrich, R. \& Slocum, T. A. (2012). Evidence-based practice: a framework for making effective decisions. Education and Treatment of Children, 35(2), 127-151. https://doi.org/10.1353/etc.2012.0013

Strupp, H.H. (1963). The outcome problem in psychotherapy. Psychotherapy, 50(1),3-11.https://doi.org/10.1037/h0094491

Strupp, H. H. (1964). The outcome problem in psychotherapy: a rejoinder. Psychotherapy: Theory, Research and Practice, 1(3), 101. https://doi.org/10.1037/h0088579

Task Force on Promotion and Dissemination of Psychological Procedures. (1995). Training in and dissemination of empirically-validated psychological treatments: report and recommendations. The Clinical Psychologist, 48(1), 3-23.

Walker, B. B., \& London, S. (2007). Novel tools and resources for evidence-based practice in psychology. Journal of Clinical Psychology, 63(7), 633-642. https:// doi.org/10.1002/jclp.20377

Wampold, B. E. (2001). The great psychotherapy debate: models, methods and findings. Mahwah: Lawrence Erlbaum.

Westen, D. I., Stirman, S. W., \& DeRubeis, R. J. (2006). Are research patients and clinical trials representative of clinical practice? In J. C. Norcross, L. E. Beutler, \& R. F. Levant (Eds.), Evidence-based practices in mental health: debate and dialogue on the fundamental questions (pp. 161-189). Washington. DC: American Psychological Association.

Woody, S. R., \& Sanderson, W. C. (1998). Manuals for empirically supported treatments: 1998 update. The Clinical Psychologist, 51(1), 17-21.

Yon, A. \& Scogin, F. (2007) Procedures for identifying evidence-based psychological treatments for older adults. Psychology and Aging, 22(1), 4-7. https://doi.org/10.1037/0882-7974.22.1.4

\section{Thiago Vinicius Monteleone}

Mestre pela Universidade São Judas Tadeu, São Paulo - SP. Brasil.

E-mail: thiagomonteleone@gmail.com

\section{Carla Witter}

Doutora pela Universidade de São Paulo. São Paulo - SP. Docente da Universidade São Judas Tadeu, São Paulo - SP.

Brasil.

E-mail: cwitter12@gmail.com

Endereço para envio de correspondência:

Universidade São Judas Tadeu. Faculdade de Ciências Biológicas e da Saúde.

Rua Taquari, 546. Mooca. CEP: 03166-000.

São Paulo - SP. Brasil.

Recebido 11/11/2015

Aprovado 14/12/2016

Received 11/11/2015

Approved 12/14/2016

Recibido 11/11/2015

Aceptado 14/12/2016 
Monteleone, T. V., \&Witter, C. (2016). Prática Baseada em Evidências em Psicologia.

Como citar: Monteleone, T.V., \&Witter, C. (2017). Prática baseada em evidências em psicologia e idosos: conceitos, estudos e perspectivas. Psicologia: Ciência e Profissão, 37(1): 48-61. http://doi.org/10.1590/1982-3703003962015

How to cite: Monteleone, T. V., \& Witter, C. (2017). Evidence-based practice in psychology and the elderly: concepts, studies and perspectives. Psicologia: Ciência e Profissão, 37(1): 48-61. http://doi.org/10.1590/1982-3703003962015

Cómo citar: Monteleone, T.V., \&Witter, C. (2017). La práctica basada en la evidencia en psicología y los adultos mayores: conceptos, estudiosy perspectivas. Psicologia:CiênciaeProfissão, 37(1):48-61.http://doi.org/10.1590/1982-3703003962015 